\title{
The importance of electron-electron bremsstrahlung for terrestrial gamma-ray flashes, electron beams and electron-positron beams
}

\author{
C Köhn ${ }^{1}$, U Ebert ${ }^{1,2}$ and A Mangiarotti ${ }^{3}$ \\ ${ }^{1}$ CWI, PO Box 94079, 1090GB Amsterdam, The Netherlands \\ ${ }^{2}$ Eindhoven University of Technology, PO Box 513, 5600MB Eindhoven, The Netherlands \\ ${ }^{3}$ Instituto de Física, Universidade de São Paulo, Brazil \\ E-mail: koehn@cwi.nl, ute.ebert@cwi.nl and alessio@if.usp.br
}

Received 27 February 2014, revised 17 April 2014

Accepted for publication 2 May 2014

Published 20 May 2014

\begin{abstract}
Thunderstorms emit terrestrial gamma-ray flashes with photon energies of up to tens of $\mathrm{MeV}$ and electron-positron beams that are created by photons with energies above $1.022 \mathrm{MeV}$. These photons are produced through the bremsstrahlung process when energetic electrons collide with air molecules. However, presently used cross sections for bremsstrahlung treat only the interaction of the electrons with the nuclei of molecules while we here include their interaction with shell electrons. We simulate the production of energetic photons by a negative stepped lightning leader, and we find that electron-electron bremsstrahlung contributes significantly, although the direct photon emission is less than from electron-nucleus bremsstrahlung. However, electron-electron bremsstrahlung also ejects shell electrons and therefore feeds the electron population above $1 \mathrm{MeV}$ significantly. We find that it hence dominates the photon spectrum of the stepped lightning leader at $10 \mathrm{MeV}$.
\end{abstract}

Keywords: electron-electron bremsstrahlung, terrestrial gamma-ray flashes, electron-positron beams, electron beams, negative stepped lightning leader

(Some figures may appear in colour only in the online journal)

\section{Introduction}

\subsection{Energetic radiation from thunderstorms and laboratory discharges}

Terrestrial gamma-ray flashes during thunderstorms were first observed by Fishman et al [1]. Meanwhile TGFs were also measured by the Reuven Ramaty High Energy Solar Spectroscopic Imager (RHESSI) [2-4], by the Fermi Gammaray Space Telescope [5] and by the Gamma-Ray Observation of Winter Thunderclouds (GROWTH) [6]. The Astrorivelatore Gamma a Immagini Leggero (AGILE) has measured quantum energies of up to $100 \mathrm{MeV}[7,8]$. Hard radiation was also observed from lightning leaders approaching the ground $[9,10]$ and in many discharge experiments performed in the laboratory under controlled conditions [11-16] where highenergy photons were created in the streamer-leader stage. It was soon understood that these flashes of energetic photons are bremsstrahlung, i.e. photons emitted when fast electrons are scattered at air molecules $[1,17]$.

Next to gamma-rays, also flashes of electrons were found to be emitted by a thunderstorm [11]; they are distinguished from photons by their dispersion relation, i.e. how they move 
in given media, and by their location since electrons as charged particles follow the geomagnetic field lines sufficiently far outside the atmosphere.

Briggs et al detected beams of positrons and electrons with the FERMI satellite over Egypt during a thunderstorm over Zambia [18]. Photons with an energy above $1 \mathrm{MeV}$ can produce pairs of electrons and positrons when scattering on the air molecules. Since positrons are charged, they will follow the geomagnetic field lines in the same manner as electrons and were thus guided from Zambia to the satellite over Egypt.

\subsection{Energetic electrons from discharges and the previously used bremsstrahlung cross sections}

The energetic photons are created through the bremsstrahlung process of energetic electrons. For the generation of energetic electrons, there are two mechanisms under debate: the older model of relativistic run-away electron avalanche in a rather homogeneous electric field inside the cloud [19-23], and the acceleration of electrons in the highly enhanced field at the tip of a streamer or leader [24-27].

For both types of models, appropriate bremsstrahlung cross sections are required to derive the photon spectrum from the electron energies. So far, geophysical researchers have used cross sections for the scattering of electrons at the nuclei of molecules. It is known [28] that the Bethe-Heitler theory for bremsstrahlung $[29,30]$ covers the energy range between $1 \mathrm{keV}$ and $1 \mathrm{GeV}$ for the small atomic numbers of air and [31] that for $Z<29$ the Bethe-Heitler theory agrees well with experimental data for the relevant energies for air molecules ( $Z=7$ for nitrogen and $Z=8$ for oxygen). Koch and Motz [32] as well as Seltzer and Berger [33] give very detailed overviews over different cross sections. They confirm that the Bethe Heitler cross section is valid for the electron scattering at light nuclei and energies between $1 \mathrm{keV}$ and $1 \mathrm{GeV}$. The tabulations by Seltzer and Berger $[33,34]$ are considered a standard reference in the field, they cover electron energies from $1 \mathrm{keV}$ to $10 \mathrm{GeV}$ and they include both electron-nucleus and electron-electron bremsstrahlung, however only the cross section singly differential in the photon energy is reported. In turn, the tabulations by Seltzer and Berger are based, for energies below or equal to $2 \mathrm{MeV}$ and for the electronnucleus bremsstrahlung, on fully numerical calculations by Pratt et al $[35,36]$. The latter are commonly considered the best available theoretical values because they employ a partial wave expansion with a self consistent procedure to describe the wavefunctions of the atomic electrons [37]. The number of partial waves necessary to accurately determine the cross section increases with energy and for this reason their tabulations stop at $2 \mathrm{MeV}$, moreover they cover only the cross section singly differential in photon energy. A detailed overview over the history of bremsstrahlung cross sections is given in [28]. We conclude that for the parameter range considered here, Bethe-Heitler is the most accurate theory available.

Up to now researchers have been using different sets of cross sections for the production of TGFs by electron-nucleus bremsstrahlung. Carlson et al [26] used the Geant 4 package with intrinsic cross sections for high atomic numbers like $Z=82$ (lead) [38]; however, we have shown in a previous paper [28] that the cross sections in Geant 4 are not appropriate for energies below $1 \mathrm{MeV}$ and for the small atomic numbers of air molecules. Xu et al [39] used a doubly differential cross section resolving the photon energy and the scattering angle of the emitted photon. They use the product ansatz introduced by Lehtinen [40] where the photon energy part is based on the Bethe-Heitler theory and the angular part is a non-quantummechanical relativistic expression by Jackson [41]. However, this ansatz is only valid for small ratios between the energy of the emitted photon and the incident electron [28]. Dwyer [22] uses the triply differential cross section by Bethe and Heitler which resolves the full geometry of the bremsstrahlung process together with the dependence on the photon energy, including an atomic form factor. In a previous paper [28], we derived a doubly differential cross section based on the Bethe-Heitler theory which relates the angle between the incident electron and emitted photon to the energy of the photon.

\section{Electron-nucleus versus electron-electron bremsstrahlung}

\subsection{Overview of mechanisms}

All the cross sections discussed above are for electron-nucleus bremsstrahlung, i.e., they parameterize the emission of photons when electrons scatter on the nuclei of atoms or molecules. However, electrons can also scatter on the shell electrons of an atom or molecule. Since electron-nucleus bremsstrahlung is proportional to $Z^{2}$ where $Z$ is the atomic number, and electronelectron bremsstrahlung is proportional to the number of shell electrons and hence to $Z$, electron-nucleus bremsstrahlung is more dominant for heavy elements than for light elements.

Electron-electron bremsstrahlung was first investigated thoroughly by Haug [42] and has been reviewed by Haug and Nakel [43]. These works treat electronelectron bremsstrahlung only between free electrons. For bremsstrahlung from shell electrons, the electron binding to the nucleus has to be included. Seltzer and Berger [33] and the ICRU [44] developed approximate expressions for bremsstrahlung from bound electrons: the ICRU [44] only for incident electrons with kinetic energy larger than $50 \mathrm{MeV}$, Seltzer and Berger [33] for energies between $1 \mathrm{keV}$ and $10 \mathrm{GeV}$ and $Z=1$ to 100 . Tessier and Kawrakow [45] extending the work by Haug and Nakel [43] calculated electron-electron bremsstrahlung cross sections for elements with $Z=1$ to 100 for incident electron energies between $1 \mathrm{keV}$ and $1 \mathrm{GeV}$, taking into account that the shell electron is initially bound and ejected during the scattering process. Thus the electronelectron bremsstrahlung process is a source of photons as well as of free electrons.

\subsection{Comparison of processes}

In this paper we use the cross sections by Bethe and Heitler [29], the electron-nucleus bremsstrahlung and the cross sections by Haug and Nakel [43] and Tessier and Kawrakow [45], which are based on previous work by Haug, 


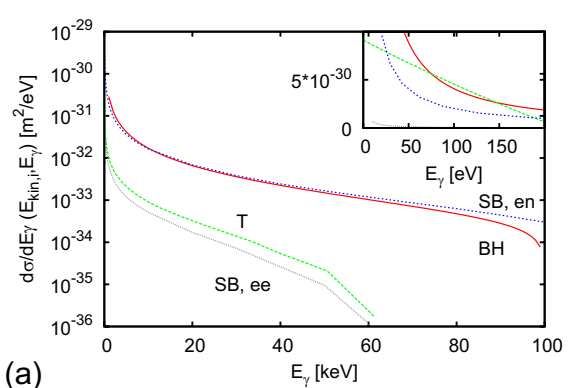

(a)

Figure 1. The differential cross section $\mathrm{d} \sigma / \mathrm{d} E_{\gamma}$ as a function of the photon energy $E_{\gamma}$ for nitrogen $(Z=7)$. The kinetic energy $E_{\mathrm{kin}, i}$ of the incident electron is $(a) 100 \mathrm{keV}$ and $(b) 10 \mathrm{MeV}$. The insets zoom into the energy region with $0 \leqslant E_{\gamma} \leqslant 200 \mathrm{eV}$; the $y$ axis of the insets is linear. The grey [33,34] and green [45] line show cross sections for electron-electron bremsstrahlung. The red [29] and blue [33,34] line show cross sections for electron-nucleus bremsstrahlung. SB denotes values by Seltzer and Berger [33,34] for electron-nucleus (en) and electron-electron (ee) bremsstrahlung; BH denotes results by Bethe and Heitler [29,30] and T by Tessier and Kawrakow [45]. (c) The total electron-electron bremsstrahlung cross section $\sigma$ per electron as a function of the energy $E_{\text {kin, } i}$ of the incident electron for the cutoffs of the photon energy at $\Lambda=1 \mathrm{eV}$ and $\Lambda=10 \mathrm{eV}$.

for electron-electron bremsstrahlung to investigate their role in the production of terrestrial gamma-ray flashes in air.

In figure 1 , we compare these two processes in terms of the differential cross section $\mathrm{d} \sigma\left(E_{\mathrm{kin}, i}, E_{\gamma}\right) / \mathrm{d} E_{\gamma}$ which is proportional to the probability that an electron with energy $E_{\text {kin }, i}$ produces a photon with an energy in the interval $\left[E_{\gamma}, E_{\gamma}+\mathrm{d} E_{\gamma}\right]$ when colliding with a nitrogen nucleus or its seven electrons. Panel (a) shows the cross sections for an incident electron energy of $100 \mathrm{keV}$, panel $(b)$ for $10 \mathrm{MeV}$. For electron-nucleus bremsstrahlung we plot the results by Bethe and Heitler (BH) [29] and Seltzer and Berger (SB,en) [33, 34]. They agree well. For electron-electron bremsstrahlung we plot calculations by Seltzer and Berger (SB,ee) $[33,34]$ and Tessier and Kawrakow (T) [45]. Note that the differential cross section for both types of bremsstrahlung decrease rapidly as a function of the photon energy. The figures show that the cross sections for electron-electron bremsstrahlung are much smaller than for electron-nucleus bremsstrahlung for photon energies above $200 \mathrm{eV}$. For electron-nucleus bremsstrahlung, both models have the same order of magnitude. Moreover the calculations by Tessier and Kawrakow [45] show a different behaviour for small photon energies. Especially for $E_{\mathrm{kin}, i}=10 \mathrm{MeV}$ the differential cross section for electron-electron bremsstrahlung according to Tessier and Kawrakow [45] becomes more important than the one for electron-nucleus bremsstrahlung for $0 \leqslant E_{\gamma} \leqslant 200 \mathrm{eV}$; this is the energy region where the differential cross section contributes most to the total cross section.

The total cross section $\sigma$ given by the integral of the differential cross section $\mathrm{d} \sigma / \mathrm{d} E_{\gamma}$ over the photon energy $E_{\gamma}$ depends on the lower limit, the cutoff $\Lambda$. In quantum electrodynamics there is an infra-red divergence in the cross section of a certain process radiating a photon whenever the same final state without that photon can be produced by another process. In the case of electron-nucleus and electron-electron bremsstrahlung, the same final state without the generation of the photon is the result of elastic electron-nucleus or elastic electron-electron (Møller) scattering, respectively. The bremsstrahlung cross section will diverge if the lowest allowed energy $\Lambda$ of the emitted photon goes to zero; therefore the cross sections are not valid for very small $\Lambda$. In our simulations we have chosen $\Lambda=1 \mathrm{eV}$. Figure 1(c) shows the total cross section for cutoffs $\Lambda=1 \mathrm{eV}$ and $\Lambda=10 \mathrm{eV}$ for electron-electron bremsstrahlung. It demonstrates that the total cross section does not change significantly if a higher cutoff is chosen; thus our results do not depend on the choice of $\Lambda$ severely.

An important fact is that the electron-electron Bremsstrahlung process does not only emit bremsstrahlung photons, but also ejects shell electrons. The electron-electron bremsstrahlung cross sections by Tessier and Kawrakow [45] and subsequently by Haug [46] show that during the emission of low-energy photons the residual energy is distributed more evenly between scattered and ejected electron than for the electron impact ionization cross sections by Kim et al [47] commonly used in discharge physics [48].

\section{Electron and photon spectra for a stepped lightning leader}

\subsection{Set-up of model and its validation}

To test the influence of electron-electron bremsstrahlung we simulate the acceleration of electrons in air consisting of $78.12 \% \mathrm{~N}_{2}, 20.95 \% \mathrm{O}_{2}$ and $0.93 \%$ Ar. We use the Monte Carlo code in three spatial dimensions that was originally designed and thoroughly tested for streamer modeling [49] and extend it to energies above $1 \mathrm{keV}$, also tracing photons, as described in [50]. Between two collisions we calculate the new position and velocity of an electron or photon using three dimensional relativistic equations of motion. For electrons, we include bremsstrahlung, elastic scattering [51-53], excitations [54], ionization [47] and attachment [54, 55] as used by [25, 27, 48]. For electron-nucleus bremsstrahlung the energy of the emitted photon is related to its scattering angle $\Theta_{i}$ by the doubly differential cross section calculated from the Bethe-Heitler theory [28,29]. For electron-electron bremsstrahlung the energy of the emitted photon is given by the spectrum as calculated by Tessier and Kawrakow [45]. The geometry of the scattered electron, the ejected electron and the emitted photon is determined by the triply differential cross section by Haug and Nakel [43]. For photons, we include Compton scattering [56], pair production [28], Rayleigh scattering [57] and 

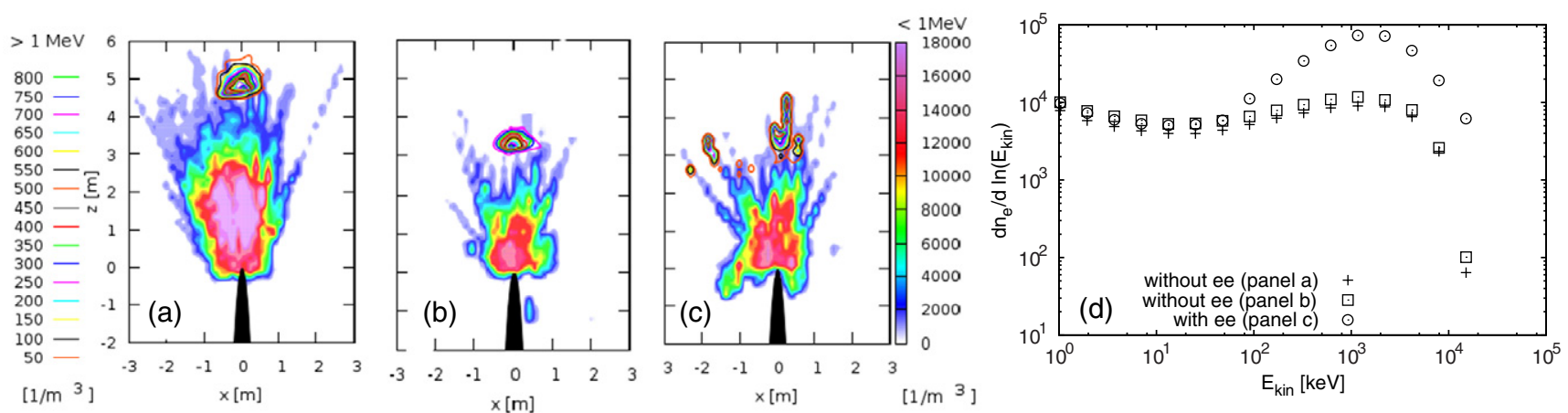

Figure 2. The electron density for different electron energies without $(a, b)$ and with $(c)$ electron-electron bremsstrahlung projected into the $x z$ plane. The lightning leader is indicated by the black region. The electrons were initially set $30 \mathrm{~cm}$ ahead of the tip. Colourlines and colourmaps represent the densities of the electrons with kinetic energies above and below $1 \mathrm{MeV}$, respectively. Panel $(a)$ and $(b)$ refer to two different stochastic Monte Carlo processes with the same distribution. $(d)$ The electron number as a function of energy for the cases shown in panels $(a),(b)$ and $(c)$.

photoionization [58] where a bremsstrahlung photon ionizes a background molecule and emits an electron. Because the equations describing the cross sections for all these processes are sometimes rather involved, extensive checks have been performed to ensure that all the interactions really occur with the appropriate probabilities. We have also checked that the final state, possibly involving the emission of another species, is correctly generated both in terms of kinematics and of probability distributions.

We investigate the production of photons in the field of a stepped lightning leader. We start with 50 electrons with an initial energy of $0.1 \mathrm{eV}$ and perform two simulations with electron-nucleus bremsstrahlung only, and one including electron-electron bremsstrahlung. Hence we are able to compare the significance of both types of bremsstrahlung.

We adopt the model by Xu et al [39] of a stepped lightning leader. We approximate the leader as a stationary ideally conducting ellipsoid with a length of $4 \mathrm{~km}$ and a curvature radius at the tip of $1 \mathrm{~cm}$ in an ambient field of $0.5 \mathrm{kV} \mathrm{cm}^{-1}$; its upper tip is at $16 \mathrm{~km}$ altitude in the terrestrial atmosphere, and the electrons are inserted at $30 \mathrm{~cm}$ ahead of the tip. The long half axis is orientated along the $z$ axis and the leader tip at $(0,0$, 0 ) of our computational domain; thus the enhancement of the electric field is highest on this axis and the motion of electrons is symmetric in $x$ and $y$. Like $\mathrm{Xu}$ et al, we neglect the electric field due to space charge effects between electrons and ions for simplicity. Therefore the low-energy spectrum is not quite physical and we only plot photon energies above $1 \mathrm{keV}$.

\subsection{Results and discussion}

Figure 2 shows the electron distributions in space without (panel $(a)$ and $(b)$ ) and with (panel $(c)$ ) electron-electron bremsstrahlung. The corresponding distributions in energy space after $24 \mathrm{~ns}$ are also displayed (panel $(d)$ ). Panel $(a)$ ) and $(b))$ are derived with different realisations of the random numbers in the Monte Carlo process while their distribution is the same. In the plots we distinguish the electrons with energies above or below $1 \mathrm{MeV}$. The fewer high-energy electrons are far ahead of the large number of low-energy electrons that would screen the electric field if space charge effects were included. But due to the spatial seperation, the highenergy electrons would hardly be affected, thus justifying our approximation. Figure $2(d)$ shows the electron number as a function of energy above $1 \mathrm{keV}$ from panels $(a),(b)$ and $(c)$ : the distributions look similar for the two cases without electronelectron bremsstrahlung, even though the spatial plots are quite different, and the electron numbers are almost the same for energies below $100 \mathrm{keV}$ in all three cases. However, there are substantially more electrons with energies above $100 \mathrm{keV}$ when electron-electron bremsstrahlung is included, as this process contributes not only to the emission of photons but also to the ejection of shell electrons. Figures 1, 2, 5 and 6 in [46] demonstrate that the residual energy $E_{\mathrm{kin}, i}-E_{\gamma}$ is shared more evenly between scattered and ejected electron than for impact ionization. Since the electron-electron bremsstrahlung process produces mostly low-energy photons, we get an enrichment of high-energy electrons which contribute to the production of high-energy bremsstrahlung photons.

Figure 3 shows the time evolution of the photon energy spectrum between $1 \mathrm{keV}$ and $10 \mathrm{MeV}$ without (panel $(a)$ ) and with (panel $(b)$ ) electron-electron bremsstrahlung. Figure 3(c) compares the photon spectra after 24 ns. Including electronelectron bremsstrahlung, the number of photons with energies above $1 \mathrm{MeV}$ is a factor 4 higher than without this process. For photons with energies above $10 \mathrm{MeV}$ this factor is 25 . The comparison also shows that the spectrum with electronelectron bremsstrahlung is slightly flatter and decreases more slowly.

\section{Conclusion}

While the past theory of terrestrial gamma-ray flashes only used the electron-nucleus bremsstrahlung process to calculate the photon spectrum, we here show that electron-electron bremsstrahlung should be included. This might be surprising at first sight since electron-electron bremsstrahlung generates less photons in the $\mathrm{MeV}$ range than the electron-nucleus process. But electron-electron bremsstrahlung contributes to the enrichment of electrons in the high-energy regime through the ejection of shell electrons. Thus there are more high-energy electrons which can produce high-energy bremsstrahlung 


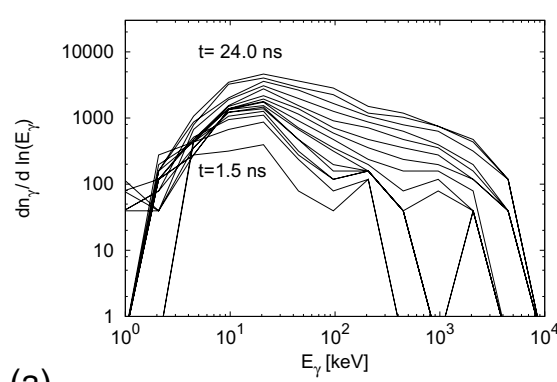

(a)

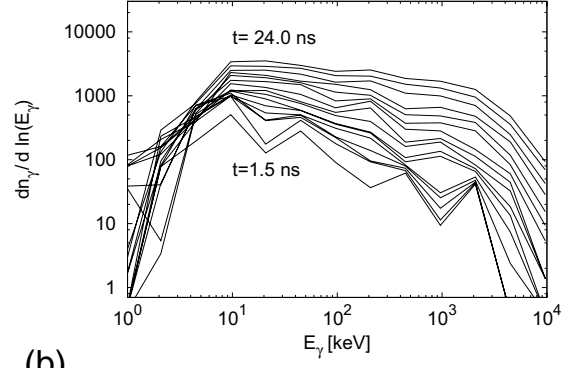

(b)

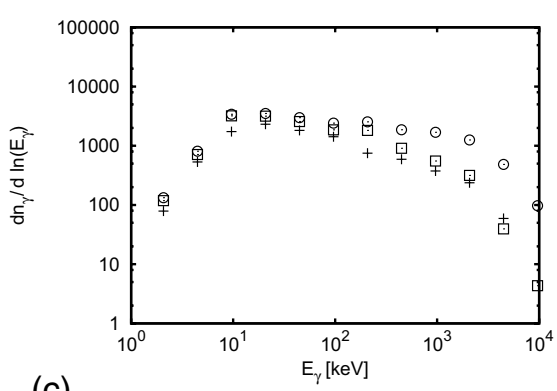

(c)

Figure 3. The time evolution of the energy resolved photon numbers produced in the field of a negative stepped leader $(a)$ without and $(b)$ with electron-electron bremsstrahlung. Every line belongs to a different time: time progresses from $1.5 \mathrm{~ns}$ for the lowest line to $24 \mathrm{~ns}$ for the highest line, in time steps of $1.5 \mathrm{~ns}$. Panel $(c)$ compares the energy dependent photon numbers with (circles) or without (crosses, boxes) electron-electron bremsstrahlung for the cases shown in figure 2. There is a substantial contribution from electron-electron bremsstrahlung at $10 \mathrm{MeV}$, with photon numbers 25 times higher than without.

photons. We have studied the electron acceleration in the field of a stepped lightning leader. In this case there are 4 times as many photons with energies above $1 \mathrm{MeV}$. There are even 25 times as many photons in the energy range between $4.8 \mathrm{MeV}$ and $10 \mathrm{MeV}$.

We conclude that electron-electron bremsstrahlung dominates the high energy spectrum of TGFs through electron ejection and hence must not be neglected. Photons with energies above $1.022 \mathrm{MeV}$ also create electron-positron pairs, therefore electron-electron bremsstrahlung is also vital for the explanation of electron-positron beams. Since electron-electron bremsstrahlung is a source of high-energy electrons, this mechanism is also important for high-energy electron beams ejected from thunderstorms.

\section{Acknowledgments}

C K acknowledges financial support by STW-project 10757, where Stichting Technische Wetenschappen (STW) is part of The Netherlands' Organization for Scientific Research NWO. A M has been partially supported by FAPESP under contract 2013/15634-5 as visiting professor at the Instituto de Física da Universidade de São Paulo.

\section{References}

[1] Fishman G J et al 1994 Discovery of intense gamma-ray flashes of atmospheric origin Science 264 1313-6

[2] Cummer S A, Zhai Y H, Hu W Y, Smith D M, Lopez L I and Stanley M A 2005 Measurements and implications of the relationship between lightning and terrestrial gamma ray flashes Geophys. Res. Lett. 32 L08811

[3] Smith D M, Lopez L I, Lin R P and Barrington-Leigh C P 2005 Terrestrial gamma-ray flashes observed up to $20 \mathrm{MeV}$ Science 307 1085-8

[4] Smith D M, Hazelton B J, Greffenstette B W, Dwyer J R, Holzworth R H and Lay E H 2010 Terrestrial gamma ray flashes correlated to storm phase and tropopause height J. Geophys. Res. 115 A00E49

[5] Briggs M S et al 2010 First results on terrestrial gamma ray flashes from the Fermi Gamma-ray Burst Monitor J. Geophys. Res. 115 A07323

[6] Tsuchiya $\mathrm{H}$ et al 2011 Long-duration gamma ray emissions from 2007 and 2008 winter thunderstorms J. Geophys. Res. 116 D09113
[7] Marisaldi M et al 2010 Detection of terrestrial gamma ray flashes up to $40 \mathrm{MeV}$ by the AGILE satellite J. Geophys. Res. 115 A00E13

[8] Tavani M et al 2011 Terrestrial gamma-ray flashes as powerful particle accelerators Phys. Rev. Lett. 106018501

[9] Moore C B, Eack K B, Aulich G D and Rison W 2001 Energetic radiation associated with lightning stepped-leaders Geophys. Res. Lett. 28 2141-4

[10] Dwyer J R et al 2005 X-ray bursts associated with leader steps in cloud-to-ground lightning Geophys. Res. Lett. 32 L01803

[11] Dwyer J R, Grefenstette B W and Smith D M 2008 High-energy electron beams launched into space by thunderstorms Geophys. Res. Lett. 35 L02815

[12] Nguyen C V, van Deursen A P J and Ebert U 2008 Multiple X-ray bursts from long discharges in air J. Phys. D: Appl. Phys. $\mathbf{4 1} 234012$

[13] Rahman M, Cooray V, Ahmad N A, Nyberg J, Rakov V A and Sharma S $2008 \mathrm{X}$-rays from $80 \mathrm{~cm}$ long sparks in air Geophys. Res. Lett. 35 L06805

[14] March V and Montanyà J 2010 Influence of the voltage-time derivative in X-ray emission from laboratory sparks Geophys. Res. Lett. 37 L19801

[15] Shao T, Zhang C, Niu Z, Yan P, Tarasenko V F, Baksht E K, Burahenko A G and Shut'ko Y V 2011 Diffuse discharge, runaway electron, and $\mathrm{X}$-ray in atmospheric pressure air in a inhomogeneous electrical field in repetitive pulsed modes Appl. Phys. Lett. 98021506

[16] Kochkin P O, Nguyen C V, van Deursen A P J and Ebert U 2012 Experimental study of hard x-rays emitted from metre-scale positive discharges in air J. Phys. D Appl. Phys 45425202

[17] Torii T, Nishijima T, Kawasaki Z I and Sugita T 2004 Downward emission of runaway electrons and Bremsstrahlung photons in thunderstorm electric fields Geophys. Res. Lett. 31 L05113

[18] Briggs M S et al 2011 Electron-positron beams from terrestrial lightning observed with Fermi GBM Geophys. Res. Lett. 38 L02808

[19] Wilson C 1925 The electric field of a thundercloud and some of its effects Proc. Phys. Soc. Lond. 37A 32D-37D

[20] Gurevich A V 1961 On the theory of runaway electrons Sov. Phys. JETP-USSR 12 904-12

[21] Dwyer J R 2003 A fundamental limit on electric fields in air Geophys. Res. Lett. 302055

[22] Dwyer J R 2007 Relativistic breakdown in planetary atmospheres Phys. Plasmas 14042901

[23] Milikh G and Roussel-Dupré R 2010 Runaway breakdown and electrical discharges in thunderstorms J. Geophys. Res. 115 A00E60 
[24] Moss G D, Pasko V P, Liu NY and Veronis G 2006 Monte Carlo model for analysis of thermal runaway electrons in streamer tips in transient luminous events and streamer zones of lightning leaders J. Geophys. Res. 111 A02307

[25] Li C, Ebert U and Hundsdorfer W 2009 3D hybrid computations for streamer discharges and production of runaway electrons J. Phys. D: Appl. Phys. 42202003

[26] Carlson B E, Lehtinen N G and Inan U S 2010 Terrestrial gamma ray flash production by active lightning leader channels J. Geophys. Res. 115 A10324

[27] Celestin S and Pasko V P 2011 Energy and fluxes of thermal runaway electrons produced by exponential growth of streamers during the stepping of lightning leaders and in transient luminous events J. Geophys. Res.-Space Phys. 116 A03315

[28] Koehn C and Ebert U 2014 Angular distribution of Bremsstrahlung photons and of positrons for calculations of terrestrial gamma-ray flashes and positron beams Atmos. Res. 135-136 432-65

[29] Bethe H A and Heitler W 1934 On the stopping of fast particles and on the creation of positive electrons Proc. Phys. Soc. London 146 83-112

[30] Heitler W 1944 The Quantum Theory of Radiation (Clarendon: Oxford University Press)

[31] Shaffer C D, Tong X M and Pratt R H 1996 Triply differential cross section and polarization correlations in electron Bremsstrahlung emission Phys. Rev. A 53 4158-63

[32] Koch H W and Motz J W 1959 Bremsstrahlung cross-section formulas and related data Rev. Mod. Phys. 31 920-56

[33] Seltzer S M and Berger M J 1985 Bremsstrahlung spectra from electron interactions with screened atomic nuclei and orbital electrons Nucl. Instrum. Methods Phys. Res. 12 95-134

[34] Seltzer S M and Berger M J 1986 Bremsstrahlung energy-spectra from electrons with kinetic energy $1 \mathrm{keV}-10 \mathrm{GeV}$ incident on screened nuclei and orbital electrons of neutral atoms with $Z=1-100$ Atom. Data Nucl. Data. 35 345-418

[35] Pratt R H, Tseng H K, Lee C M, Kissel, L MacCallum C and Riley M 1977 Bremsstrahlung energy spectra from electrons of kinetic energy $1 \mathrm{keV}<T_{1}<2000 \mathrm{keV}$ incident on neutral atoms $2<Z<29$ Atom. Data Nucl. Data 20 175-209

[36] Pratt R H, Tseng H K, Lee C M, Kissel L, MacCallum C and Riley M 1981 Errata of Bremsstrahlung energy spectra from electrons of kinetic energy $1 \mathrm{keV}<T_{1}<2000 \mathrm{keV}$ incident on neutral atoms $2<Z<29$ Arom. Data Nucl. Data 26 477-81

[37] Tseng H K and Pratt R H 1971 Exact screened calculations of atomic-field Bremsstrahlung Phys. Rev. A 3 100-15

[38] Agostinelli S et al 2003 G4-a simulation toolkit Nucl. Instrum. Methods Phys. Res., Sect. A 506 250-303
[39] Xu W, Celestin S and Pasko V P 2012 Source altitudes of terrestrial gamma-ray flashes produced by lightning leaders Geophys. Res. Lett. 39 L08801

[40] Lehtinen N G 2000 Relativistic runaway electrons above thunderstorms Dissertation (Stanford University)

[41] Jackson J D 1975 Classical Electrodynamics (New York: Wiley)

[42] Haug E 1975 Bremsstrahlung and pair production in field of free electrons Z. Naturforschung 30 1099-113

[43] Haug E and Nakel W 2004 The Elementary Process of Bremsstrahlung vol 73 (Singapore: World Scientific)

[44] ICRU 1984 Stopping powers for electrons and positrons ICRU Report no. 37

[45] Tessier F and Kawrakow I 2007 Calculation of the electron-electron bremsstrahlung cross-section in the field of atomic electrons NIM. Phys. Res. B 266 625-34

[46] Haug E 2008 Electron-electron bremsstrahlung for bound target electrons Eur. Phys. J. D 49 193-9

[47] Kim Y K, Santos J P and Parente F 2000 Extension of the binary-encounter-dipole model to relativistic incident electrons Phys. Rev. A 62052710

[48] Celestin S and Pasko V P 2010 Soft collisions in relativistic runaway electron avalanches J. Phys. D: Appl. Phys 43315206

[49] Li C, Teunissen J, Nool M, Hundsdorfer W and Ebert U 2012 A comparison of 3D fluid, particle and hybrid model for negative streamers Plasma Sour. Sci. Technol. 21055019

[50] Koehn C and Ebert U 2014 The structure of ionization showers in air generated by electrons with $1 \mathrm{MeV}$ energy or less revised for Plasma Sour. Sci. Technol.

[51] Jacob J H 1973 Multiple electron-scattering through a slab Phys. Rev. A 8 226-35

[52] Phelps A V and Pitchford L C 1985 Anisotropic scattering of electrons by $\mathrm{N}_{2}$ and its effect on electron transport Phys. Rev. A 31 2932-49

[53] Phelps A V 1985 JILA Information Center Report No. 28 (University of Colorado)

[54] Lawton S A and Phelps A V 1978 Excitation of $b^{1} \Sigma_{\mathrm{g}}^{+}$state of $\mathrm{O}_{2}$ by low-energy electrons J. Chem. Phys. $691055-68$

[55] Pancheshnyi S, Eismann B, Hagelaar G J M and Pitchford L C 2008 Computer code ZDPlasKin www.zdplaskin.laplace. univ-tlse.fr (University of Toulouse, LAPLACE, CNRS-UPS-INP, Toulouse, France)

[56] Peskin M E and Schroeder D V 1995 An Introduction to Quantum Field Theory (Boston, MA: Addision-Wesley)

[57] Shardanand and Prasad Rao A D 1977 Absolute Rayleigh scattering cross sections of gases and freons of stratospheric interest in the visible and ultraviolet regions NASA Technical Note

[58] Photon and Electron Interaction Data 1997 www-nds.iaea.org/epd197/ 